\title{
Highly Enantioselective Azadiene Diels-Alder Reactions Catalyzed by Chiral $N$-Heterocyclic Carbenes
}

\author{
Ming He, Justin R. Struble, and Jeffrey W. Bode* \\ Department of Chemistry and Biochemistry, University of California, \\ Santa Barbara, CA 93106-9510
}

\section{Supporting Information}

General Methods. All reactions utilizing air- or moisture-sensitive reagents were performed in dried glassware under an atmosphere of dry argon. Chlorobenzene and $\mathrm{CH}_{2} \mathrm{Cl}_{2}$ were distilled over $\mathrm{CaH}_{2}$. Toluene and THF were dried by passage over activated alumina under $\mathrm{Ar}$ atmosphere. All aldehydes were purified by distillation prior to use. Diisopropylethylamine (DIPEA) was distilled from KOH. Other reagents were used without further purification. Thin layer chromatography (TLC) was performed on Merck precoated plates (silica gel $60 \mathrm{~F}_{254}$, Art $5715,0.25 \mathrm{~mm}$ ) and were visualized by fluorescence quenching under UV light or by staining with phosphomolybdic acid. Silica-gel preparative thin-layer chromatography (PTLC) was performed using plates prepared from Merck Kieselgel $60 \mathrm{PF}_{254}$ (Art 7747). Column chromatography was performed on E. Merck Silica Gel 60 (230-400 Mesh) using a forced flow of 0.5-1.0 bar. ${ }^{1} \mathrm{H}$ NMR (400 MHz) and ${ }^{13} \mathrm{C}$ NMR $(100 \mathrm{MHz})$ were measured on a Varian Unity 400 spectrometer. Chemical shifts are expressed in parts per million (PPM) downfield from residual solvent peaks and coupling constants are reported as Hertz (Hz). Splitting patterns are indicated as follows: br, broad; s, singlet; d, doublet; t, triplet; q, quartet; m, multiplet. Infrared (IR) spectra were recorded on a JASCO FT/IR-430 spectrophotometer and are reported as wavenumber $\left(\mathrm{cm}^{-1}\right)$. Optical rotations were measured on a Jasco DIP-1000 polarimeter operating at the sodium $\mathrm{D}$ line with a $100 \mathrm{~mm}$ path length cell, and are reported as follows: $[\alpha]^{\mathrm{T}}$ (concentration $(\mathrm{g} / 100 \mathrm{ml})$, solvent).

HPLC Conditions. Column, Diacel Chiralpak AS-H, (4.6 x 250mm) Eluent: hexanes/i-PrOH. Flow Rate 1.0 mL/min. Detection: $254 \mathrm{~nm}$. Column, Diacel Chiralpak AD-H, (4.6 x250mm) Eluent: hexanes/iPrOH. Flow Rate $1.0 \mathrm{~mL} / \mathrm{min}$. Detection: $254 \mathrm{~nm}$. Column, Diacel Chiralpak OD-H, (4.6 x250mm) Eluent: hexanes/iPrOH. Flow Rate 1.0 mL/min. Detection: 254 nm. 


\section{General procedure for NHC-catalyzed, enantioselective azadiene Diels-Alder reactions.}

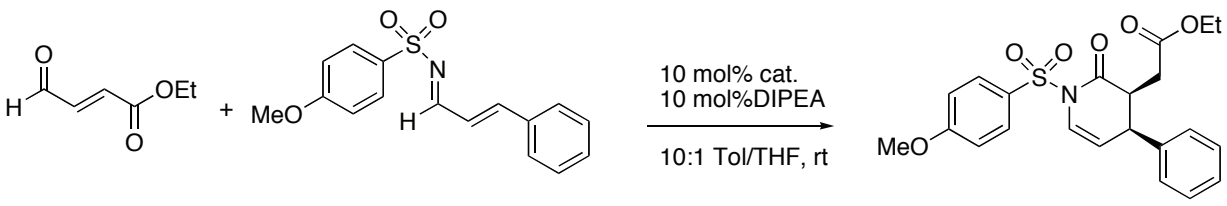

The reaction of ethyl trans-4-oxo-2-butenoate ${ }^{1}$ and $\operatorname{trans}^{-N} \mathrm{~N}$-(4-methoxybenzenesulfonyl)-3phenyprop-2-ene-1-imine is representative: Into an oven dried $2.0 \mathrm{~mL}$ vial was added the enal (6.5 ml, $0.054 \mathrm{mmol}, 1.1$ equiv), imine (14.7 mg, $0.049 \mathrm{mmol}, 1.0$ equiv) and triazolium salt 9 (1.8mg, 0.005 mmol, 0.10 equiv). The vial was crimped with a Teflon-lined crimp seal. To this mixture was added $1.0 \mathrm{~mL} \mathrm{10:1} \mathrm{toluene/THF}(0.05 \mathrm{M})$, followed by diisopropylethylamine $(0.8$ $\mu \mathrm{L}, 0.005 \mathrm{mmol}, 0.10$ equiv). The resulting solution was stirred at room temperature for $23 \mathrm{~h}$. The reaction mixture was concentrated under reduced pressure, and the residue was purified by PTLC (2:1 hexane/EtOAc) to afford the dihydropyridinone product as a white solid (19.8 $\mathrm{mg}$, $90 \%$ yield).

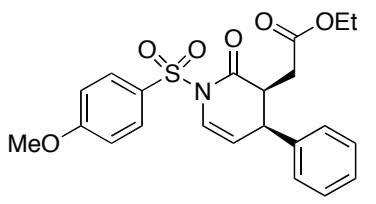

Ethyl 2-((3S,4S)-1-(4-methoxyphenylsulfonyl)-2-oxo-4-phenyl-1,2,3,4-tetrahydropyridin-3yl)acetate (Table 2, entry 1). Prepared according to the general procedure from ethyl trans-4oxo-2-butenoate and trans- $N$-(4-methoxybenzenesulfonyl)-3-phenyprop-2-ene-1-imine using 10 mol $\% 9$ as the catalyst in $90 \%$ yield as a white solid. $[\alpha]_{\mathrm{D}}{ }^{20}\left(\mathrm{c} 1.19, \mathrm{CHCl}_{3}\right)=+105.2 ; \mathrm{mp}=$ 111-113 ${ }^{\circ} \mathrm{C} ;{ }^{1} \mathrm{H}$ NMR (400 MHz, $\mathrm{CDCl}_{3}$ ) $\delta 8.04$ (dd, 2H, J=7.0, 2.1 Hz), 7.16-7.12 (m, 2H), 7.06-6.99 (m, 4H), $6.58(\mathrm{dd}, 2 \mathrm{H}, J=8.0,1.1 \mathrm{~Hz}), 5.60(\mathrm{dd}, 1 \mathrm{H}, J=8.0,6.5 \mathrm{~Hz}), 4.18-4.08(\mathrm{~m}$, $2 \mathrm{H}), 3.92(\mathrm{~s}, 3 \mathrm{H}), 3.65(\mathrm{t}, 1 \mathrm{H}, J=6.9 \mathrm{~Hz}), 3.55-3.53(\mathrm{~m}, 1 \mathrm{H}), 2.57(\mathrm{dd}, 1 \mathrm{H}, J=17.3,6.1 \mathrm{~Hz})$, $1.94(\mathrm{dd}, 1 \mathrm{H}, J=17.3,7.5 \mathrm{~Hz}), 1.21(\mathrm{t}, 3 \mathrm{H}, J=7.0 \mathrm{~Hz}) ;{ }^{13} \mathrm{C}$ NMR $\left(100 \mathrm{MHz}, \mathrm{CDCl}_{3}\right) \delta 171.7$, 169.0, 164.3, 136.8, 131.4, 129.2, 128.9, 127.9, 127.9, 124.6, 114.3, 113.6, 61.0, 56.0, 44.1, 42.0, 31.6, 14.3; IR (thin film) v 3086, 2982, 1720, 1594, 1496, 1454, 1406, 1367, 1264, 1132, 1091,

(1) Lancaster Synthesis (Alfa Aesar) Cat. No. 18481 (25 g/\$50.97). 
$1026 \mathrm{~cm}^{-1}$; HRMS (ESI) calcd for $\mathrm{C}_{22} \mathrm{H}_{23} \mathrm{NO}_{6} \mathrm{~S}[\mathrm{M}+\mathrm{Na}]^{+} 452.1138$, found 452.1160; >99\% ee $(3 R, 4 R)$-isomer as determined by HPLC (AS-H, 9:1 hexanes/i-PrOH $), t_{\mathrm{r}}(3 S, 4 S)=53.9 \mathrm{~min}$, $t_{\mathrm{r}}(3 R, 4 R)=39.2$ min. For the $(3 R, 4 R)$ enantiomer $($ Table 2 , entry 2$),[\alpha]_{\mathrm{D}}{ }^{20}\left(\mathrm{c} 0.98, \mathrm{CHCl}_{3}\right)=-$ 112.7.

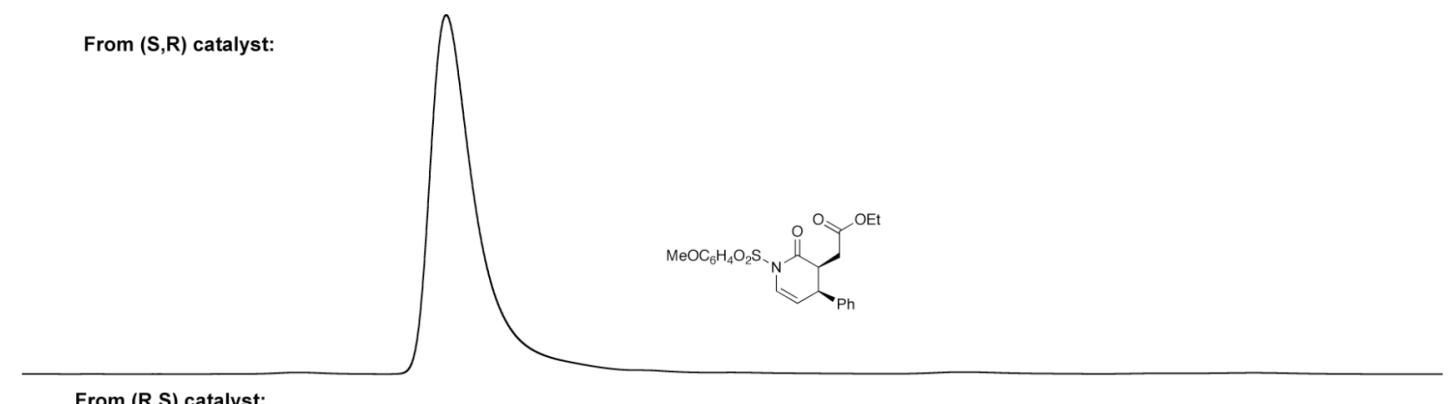

From $(\mathbf{R}, \mathbf{S})$ catalyst:
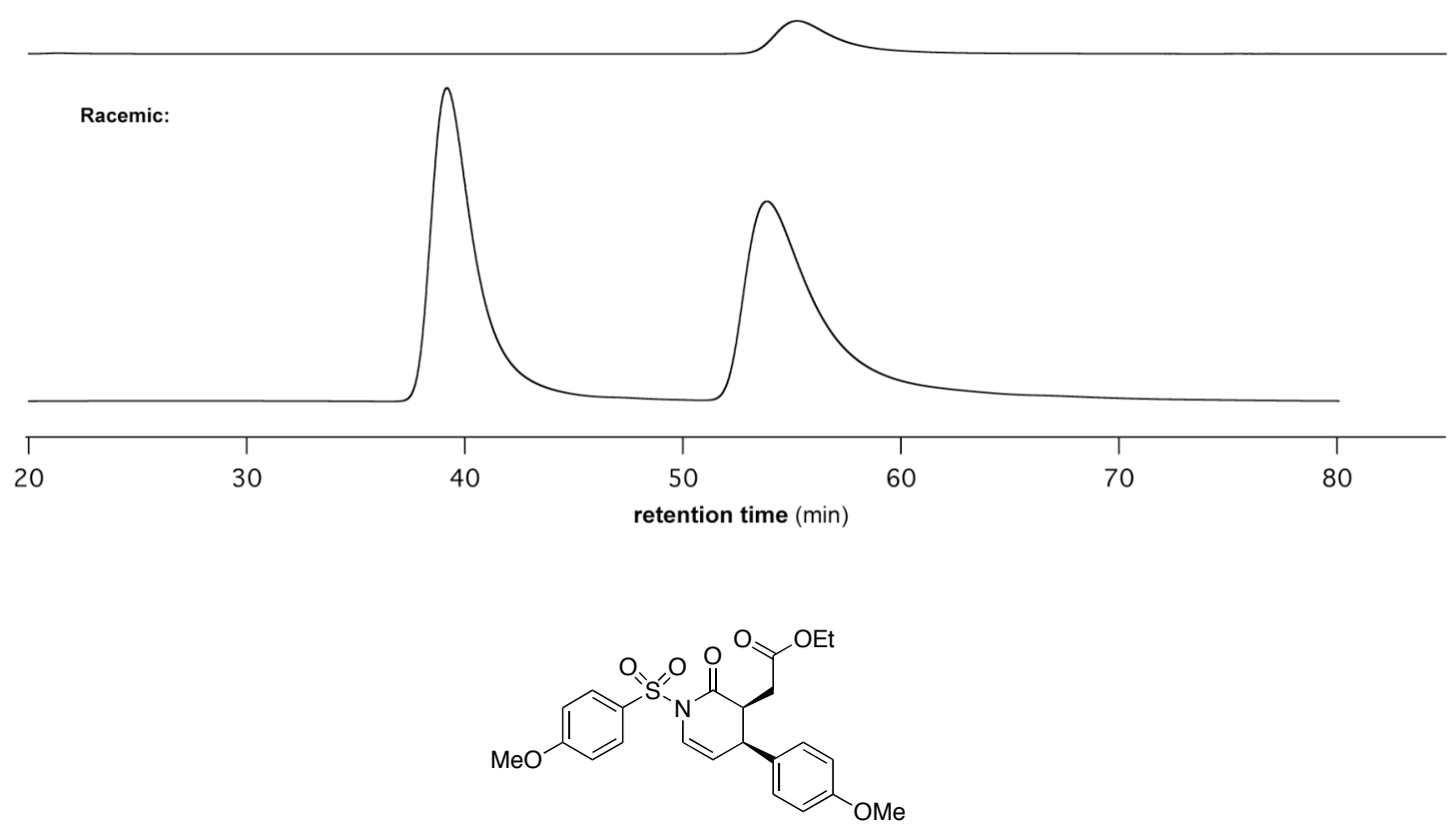

Ethyl 2-((3S,4S)-4-(4-methoxyphenyl)-1-(4-methoxyphenylsulfonyl)-2-oxo-1,2,3,4-tetrahydropyridin-3-yl)acetate (Table 2, entry 3). Prepared according to the general procedure from ethyl trans-4-oxo-2-butenoate and trans- $N$-(4-methoxybenzenesulfonyl)-3-(4methoxyphenyl)prop-2-en-1-imine using $10 \mathrm{~mol} \% \mathbf{9}$ as the catalyst in $81 \%$ yield as a yellow solid. $[\alpha]_{\mathrm{D}}{ }^{20}\left(\mathrm{c} 1.04, \mathrm{CHCl}_{3}\right)=+143.6 ; \mathrm{mp}=107-110{ }^{\circ} \mathrm{C} ;{ }^{1} \mathrm{H} \mathrm{NMR}\left(400 \mathrm{MHz}, \mathrm{CDCl}_{3}\right) \delta 8.03$ $(\mathrm{dd}, 2 \mathrm{H}, J=7.1,1.8 \mathrm{~Hz}), 7.13(\mathrm{~d}, 1 \mathrm{H}, J=8.1 \mathrm{~Hz}), 7.05(\mathrm{dd}, 2 \mathrm{H}, J=7.1,1.8 \mathrm{~Hz}), 6.55-6.48$ (m, 4H), $5.59(\mathrm{dd}, 1 \mathrm{H}, J=8.0,6.6 \mathrm{~Hz}), 4.16-4.09(\mathrm{~m}, 2 \mathrm{H}), 3.92(\mathrm{~s}, 3 \mathrm{H}), 3.71(\mathrm{~s}, 3 \mathrm{H}), 3.60(\mathrm{t}, 1 \mathrm{H}, J$ 
= 6.9 Hz), 3.52-3.47 (m, 1H), $2.58(\mathrm{dd}, 1 \mathrm{H}, J=17.2,6.0 \mathrm{~Hz}), 1.95(\mathrm{dd}, 1 \mathrm{H}, J=17.2,7.7 \mathrm{~Hz})$, $1.22(\mathrm{t}, 3 \mathrm{H}, J=7.0 \mathrm{~Hz}) ;{ }^{13} \mathrm{C}$ NMR $\left(100 \mathrm{MHz}, \mathrm{CDCl}_{3}\right) \delta 171.8,169.1,164.3,159.2,131.4,129.2$, $128.5,124.3,114.3,114.3,113.9,113.6,61.0,56.0,55.3,44.3,41.2,31.5,14.4$; IR (thin film) $v$ 3055, 2840, 1720, 1647, 1511, 1498, 1463, 1442, 1366, 1263, 1167, 1090, $1028 \mathrm{~cm}^{-1}$; HRMS (ESI) calcd for $\mathrm{C}_{23} \mathrm{H}_{25} \mathrm{NO}_{7} \mathrm{~S}[\mathrm{M}+\mathrm{Na}]^{+} 482.1243$, found 482.1253; $>99 \%$ ee $(3 S, 4 S)$-isomer as determined by HPLC (AS-H, 4:1 hexanes/i-PrOH), $t_{\mathrm{r}}(3 S, 4 S)=49.3 \mathrm{~min}, t_{\mathrm{r}}(3 R, 4 R)=29.6 \mathrm{~min}$.

From $(\mathbf{R}, \mathbf{S})$ Catalyst:
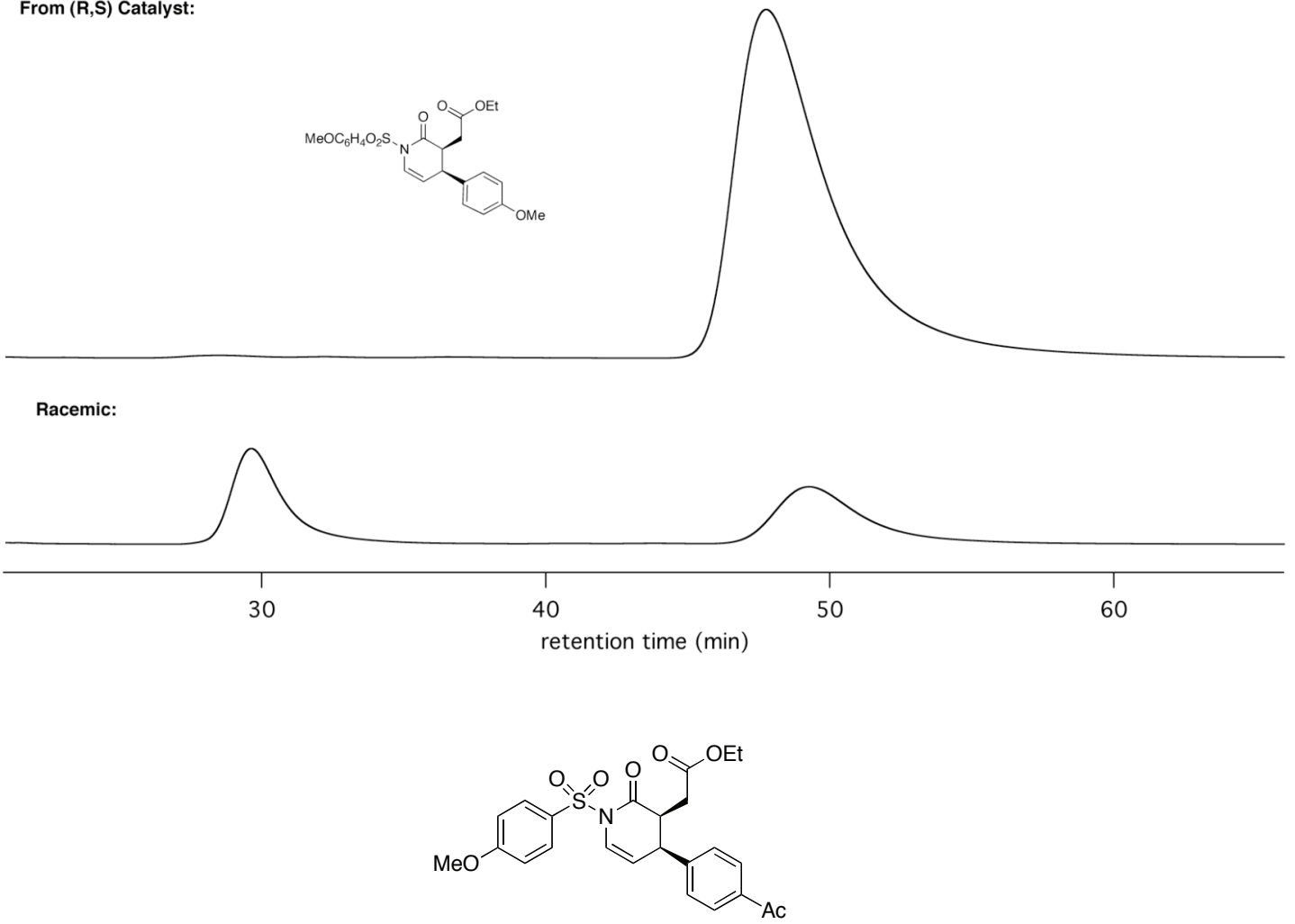

Ethyl 2-((3S,4S)-4-(4-acetylphenyl)-1-(4-methoxyphenylsulfonyl)-2-oxo-1,2,3,4-tetrahydropyridin-3-yl)acetate (Table 2, entry 4). Prepared according to the general procedure from ethyl trans-4-oxo-2-butenoate and trans- $N$-(4-methoxybenzenesulfonyl)-1-(4-(3-iminoprop-1enyl)phenyl)ethanone using $10 \mathrm{~mol} \% \mathbf{9}$ as the catalyst in $55 \%$ yield as a yellow oil. $[\alpha]_{\mathrm{D}}{ }^{20}(\mathrm{c}$ 1.22, $\left.\mathrm{CHCl}_{3}\right)=+168.2 ;{ }^{1} \mathrm{H} \mathrm{NMR}\left(400 \mathrm{MHz}, \mathrm{CDCl}_{3}\right) \delta 8.04(\mathrm{dd}, 2 \mathrm{H}, J=6.9,2.1 \mathrm{~Hz}), 7.59(\mathrm{dd}$, $2 \mathrm{H}, J=6.5,1.9 \mathrm{~Hz}), 7.19(\mathrm{~d}, 1 \mathrm{H}, J=8.0 \mathrm{~Hz}), 7.07(\mathrm{dd}, 2 \mathrm{H}, J=6.9,2.1 \mathrm{~Hz}), 6.69(\mathrm{dd}, 2 \mathrm{H}, J=$ 
6.6, 1.7 Hz), 5.59 (dd, 1H, $J=8.0,6.6 \mathrm{~Hz}), 4.14-4.11(\mathrm{~m}, 2 \mathrm{H}), 3.95(\mathrm{~s}, 3 \mathrm{H}), 3.74(\mathrm{t}, 1 \mathrm{H}, J=6.9$ Hz), 3.58- $3.53(\mathrm{~m}, 1 \mathrm{H}), 2.58(\mathrm{dd}, 1 \mathrm{H}, J=17.4,6.0 \mathrm{~Hz}), 2.52$ (s, 3H), $1.90(\mathrm{dd}, 1 \mathrm{H}, J=17.4,7.8$ $\mathrm{Hz}), 1.22$ (t, 3H, $J=7.2 \mathrm{~Hz}) ;{ }^{13} \mathrm{C} \mathrm{NMR}\left(100 \mathrm{MHz}, \mathrm{CDCl}_{3}\right) \delta 197.5,171.5,168.6,164.5,142.3$, 136.6, 131.4, 128.9, 128.2, 125.3, 114.3, 112.7, 94.6, 61.1, 56.1, 43.9, 41.7, 31.4, 26.7, 14.3; IR (thin film) $v$ 3020, 2983, 2842, 1723, 1682, 1594, 1497, 1363, 1267, 1167, 1091, $1024 \mathrm{~cm}^{-1}$; HRMS (ESI) calcd for $\mathrm{C}_{24} \mathrm{H}_{25} \mathrm{NO}_{7} \mathrm{~S}[\mathrm{M}+\mathrm{Na}]^{+} 494.1243$, found 494.1265; >99\% ee $(3 S, 4 S)$ isomer as determined by HPLC $(\mathrm{OD}-\mathrm{H}, 4: 1$ hexanes/i-PrOH$), t_{\mathrm{r}}(3 S, 4 S)=28.6 \min , t_{\mathrm{r}}(3 R, 4 R)=$ $35.1 \mathrm{~min}$.
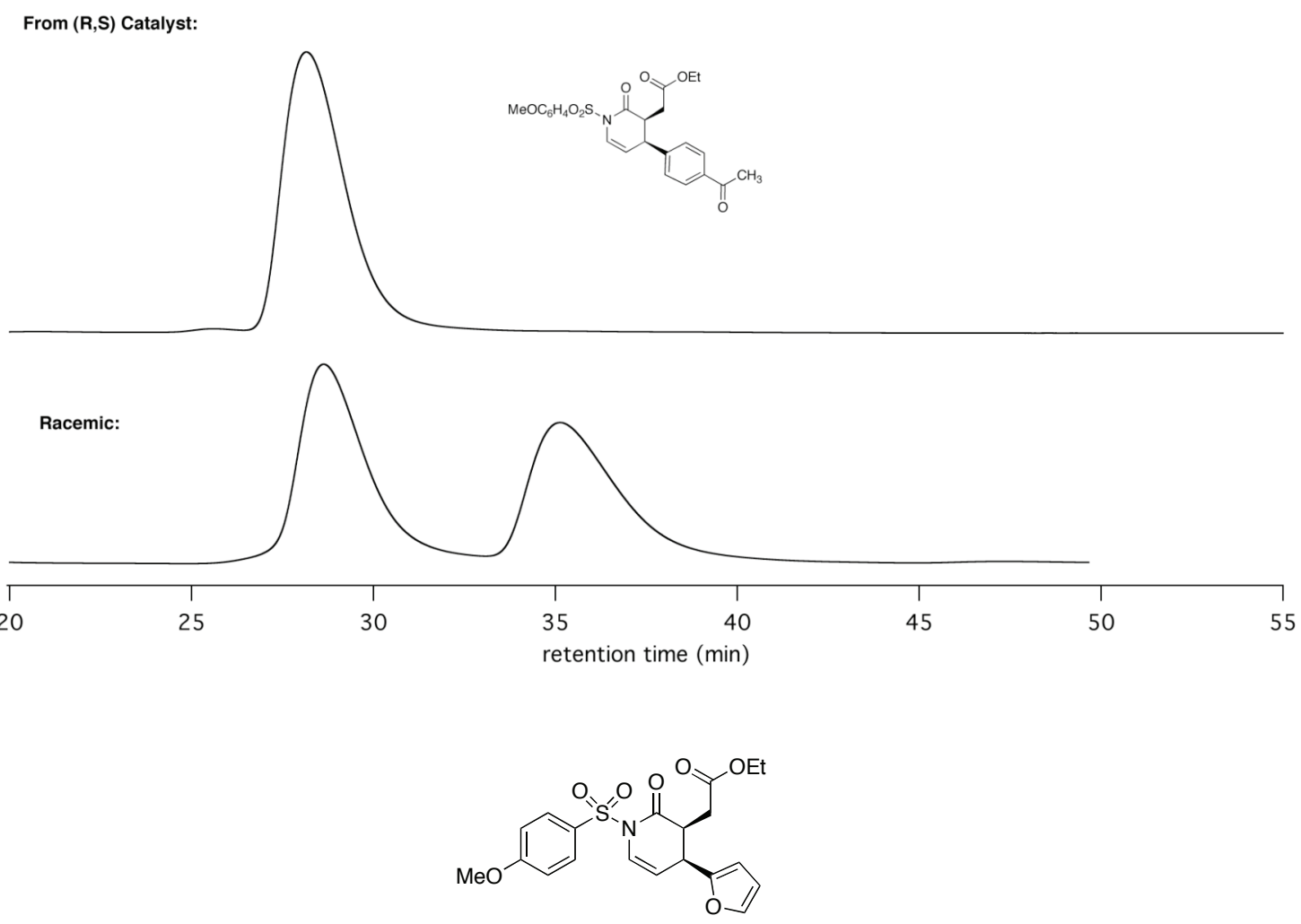

Ethyl 2-((3S,4S)-4-(furan-2-yl)-1-(4-methoxyphenylsulfonyl)-2-oxo-1,2,3,4-tetrahydropyridin-3-yl)acetate (Table 2, entry 5). Prepared according to the general procedure from ethyl trans-4-oxo-2-butenoate and trans- $N$-(4-methoxybenzenesulfonyl)-3-(furan-2-yl)prop-2-en-1imine using $10 \mathrm{~mol} \% \mathbf{9}$ as the catalyst in $71 \%$ yield as a yellow solid. $[\alpha]_{\mathrm{D}}^{20}\left(\mathrm{c} 1.58, \mathrm{CHCl}_{3}\right)=$ +125.7; mp = 98-100 ${ }^{\circ} \mathrm{C} ;{ }^{1} \mathrm{H}$ NMR $\left(400 \mathrm{MHz} \mathrm{CDCl}_{3}\right) \delta 7.98(\mathrm{dd}, 2 \mathrm{H}, J=7.0,1.9 \mathrm{~Hz}), 7.12$ (d, 
$2 \mathrm{H}, J=8.0 \mathrm{~Hz}), 7.00(\mathrm{dd}, 2 \mathrm{H}, J=7.0,1.9 \mathrm{~Hz}), 6.87(\mathrm{~d}, 1 \mathrm{H}, J=1.8 \mathrm{~Hz}), 6.11(\mathrm{dd}, 1 \mathrm{H}, J=3.2$, $1.8 \mathrm{~Hz}), 5.82(\mathrm{~d}, 1 \mathrm{H}, J=3.2 \mathrm{~Hz}), 5.51(\mathrm{dd}, 1 \mathrm{H}, J=8.0,6.8 \mathrm{~Hz}), 4.15-4.10(\mathrm{~m}, 2 \mathrm{H}), 3.90$ (s, $3 \mathrm{H}), 3.75(\mathrm{t}, 1 \mathrm{H}, J=6.7 \mathrm{~Hz}), 3.45-3.40(\mathrm{~m}, 1 \mathrm{H}), 2.72(\mathrm{dd}, 1 \mathrm{H}, J=17.3,6.0 \mathrm{~Hz}), 2.52(\mathrm{~s}, 3 \mathrm{H})$, $2.04(\mathrm{dd}, 1 \mathrm{H}, J=17.3,7.7 \mathrm{~Hz}), 1.22(\mathrm{t}, 3 \mathrm{H}, J=7.0 \mathrm{~Hz}) ;{ }^{13} \mathrm{C} \mathrm{NMR}\left(100 \mathrm{MHz}, \mathrm{CDCl}_{3}\right) \delta 171.7$, 168.7, 164.2, 142.6, 131.4, 129.6, 125.7, 114.1, 110.4, 108.0, 61.0, 56.0, 43.2, 35.1, 31.5, 14.3; IR (thin film) v 3114, 2982, 2843, 1724, 1595, 1498, 1363, 1263, 1167, 1133, 1092, $1025 \mathrm{~cm}^{-1}$; HRMS (ESI) calcd for $\mathrm{C}_{20} \mathrm{H}_{21} \mathrm{NO}_{7} \mathrm{~S}[\mathrm{M}+\mathrm{Na}]^{+} 494.1243$, found 494.1265; >99\% ee $(3 S, 4 S)$ isomer as determined by HPLC $(\mathrm{AD}-\mathrm{H}, 9: 1$ hexanes/i-PrOH $), t_{\mathrm{r}}(3 S, 4 S)=43.7 \mathrm{~min}, t_{\mathrm{r}}(3 R, 4 R)=$ $27.4 \mathrm{~min}$.

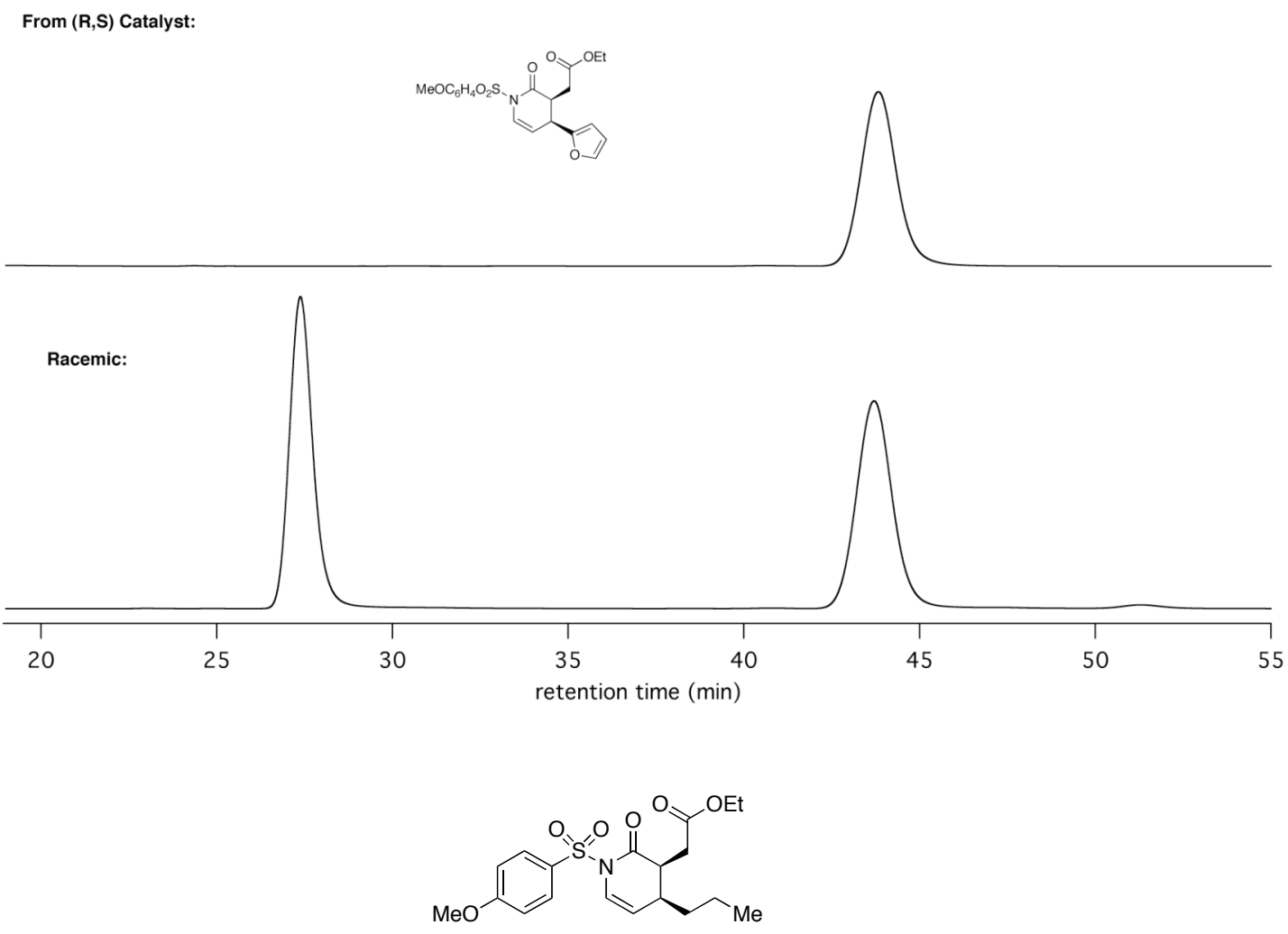

Ethyl 2-((3S,4S)-1-(4-methoxyphenylsulfonyl)-2-oxo-4-propyl-1,2,3,4-tetrahydropyridin-3yl)acetate (Table 1, entry 6). Prepared according to the general procedure from ethyl trans-4oxo-2-butenoate and trans- $N$-(4-methoxybenzenesulfonyl)-hex-2-en-1-imine using 10 mol \% 9 as the catalyst in $58 \%$ yield as a colorless oil. $[\alpha]_{\mathrm{D}}{ }^{20}\left(\mathrm{c} 0.64, \mathrm{CHCl}_{3}\right)=-17.3 ;{ }^{1} \mathrm{H}$ NMR $(400$ 
$\left.\mathrm{MHz}, \mathrm{CDCl}_{3}\right) \delta 7.92(\mathrm{dd}, 2 \mathrm{H}, J=6.9,2.0 \mathrm{~Hz}), 6.97(\mathrm{dd}, 2 \mathrm{H}, J=6.9,2.0 \mathrm{~Hz}), 5.53(\mathrm{dd}, 1 \mathrm{H}, J=$ 8.0, 6.6 Hz), 4.13- 4.11 (m, 2H ), 3.87 (s, 3H), 3.24 (q, 1H, J = 6.3 Hz), 2.82 (dd, 1H, $J=16.6$, $7.2 \mathrm{~Hz}), 2.41-2.38(\mathrm{~m}, 1 \mathrm{H}), 2.24(\mathrm{dd}, 1 \mathrm{H}, J=16.6,7.0 \mathrm{~Hz}), 1.22(\mathrm{t}, 3 \mathrm{H}, J=7.2 \mathrm{~Hz}), 1.25-1.02$ $(\mathrm{m}, 4 \mathrm{H}), 0.73(\mathrm{t}, 3 \mathrm{H}, J=7.2 \mathrm{~Hz}) ;{ }^{13} \mathrm{C} \mathrm{NMR}\left(100 \mathrm{MHz}, \mathrm{CDCl}_{3}\right) \delta 171.7,170.2,164.2,131.1$, $129.5,124.2,114.9,114.1,61.0,55.9,43.4,34.5,31.6,31.4,19.3,14.3,14.2$; IR (thin film) $v$ 3046, 1723, 1595, 1506, 1418, 1358, 1271, $1133 \mathrm{~cm}^{-1}$; HRMS (ESI) calcd for $\mathrm{C}_{19} \mathrm{H}_{25} \mathrm{NO}_{6} \mathrm{~S}$ $[\mathrm{M}+\mathrm{Na}]^{+} 418.1294$, found $418.1287 ;>99 \%$ ee $(3 S, 4 S)$-isomer as determined by HPLC (AS-H, 9:1 hexanes/i-PrOH $), t_{\mathrm{r}}(3 S, 4 S)=55.0 \mathrm{~min}, t_{\mathrm{r}}(3 R, 4 R)=28.2 \mathrm{~min}$.

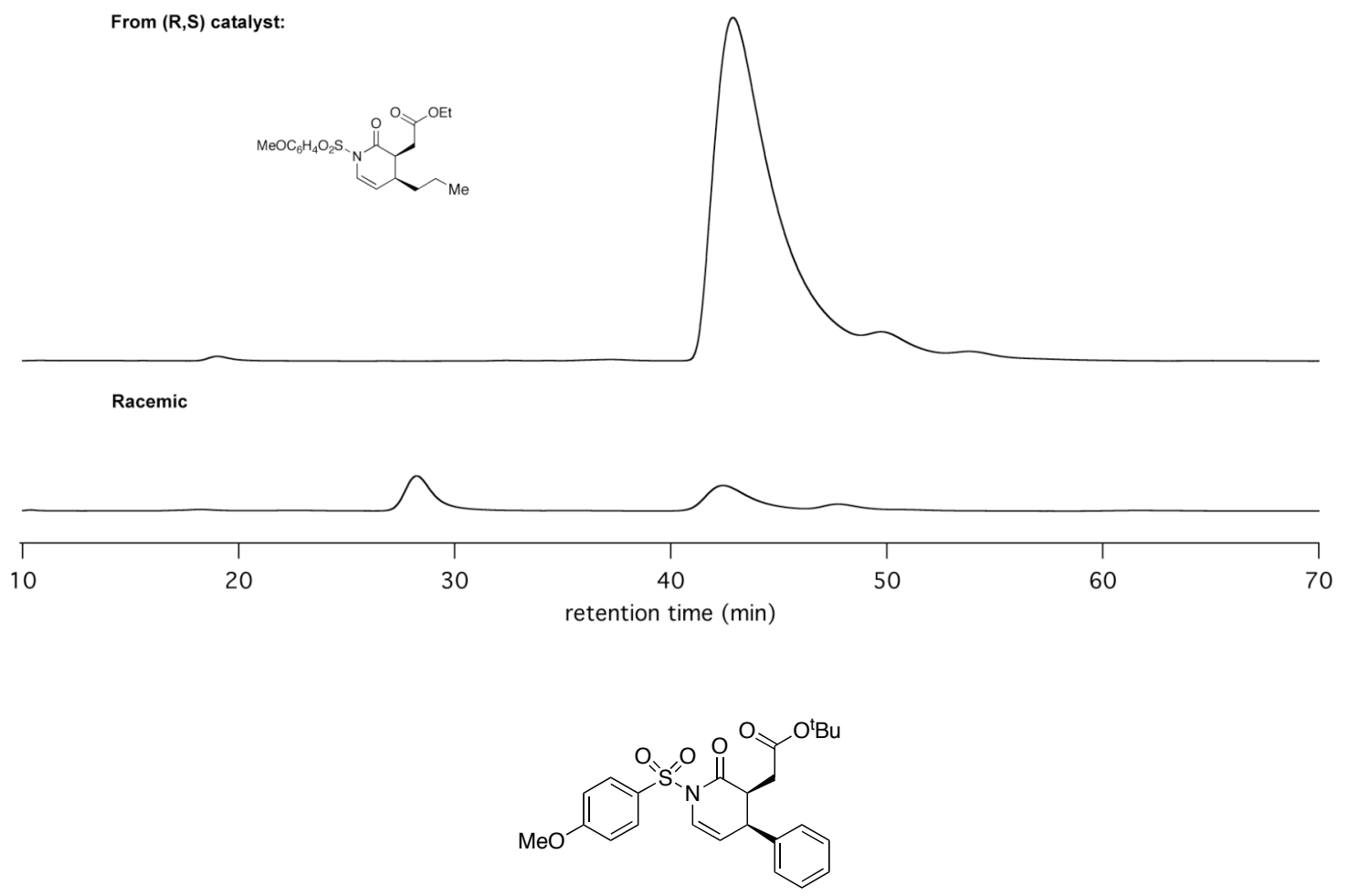

tert-Butyl 2-((3S,4S)-1-(4-methoxyphenylsulfonyl)-2-oxo-4-phenyl-1,2,3,4-tetrahydropyridin-3-yl)acetate (Table 3, entry 1). Prepared according to the general procedure from tertbutyl trans-4-oxo-2-butenoate and trans- $N$-(4-methoxybenzenesulfonyl)-3-phenylprop-2-en-1imine using $10 \mathrm{~mol} \% 9$ as the catalyst in $70 \%$ yield white solid. $[\alpha]_{\mathrm{D}}{ }^{20}\left(\mathrm{c} 1.07, \mathrm{CHCl}_{3}\right)=+90.4$; $\mathrm{mp}=139-142{ }^{\circ} \mathrm{C} ;{ }^{1} \mathrm{H} \mathrm{NMR}\left(400 \mathrm{MHz}, \mathrm{CDCl}_{3}\right) \delta 8.03(\mathrm{dd}, 2 \mathrm{H}, J=6.9,2.1 \mathrm{~Hz}), 7.16-7.13(\mathrm{~m}$, 
2H), 7.05- $7.00(\mathrm{~m}, 4 \mathrm{H}), 6.60(\mathrm{dd}, 2 \mathrm{H}, J=8.2,1.0 \mathrm{~Hz}), 5.59(\mathrm{dd}, 1 \mathrm{H}, J=8.1,6.6 \mathrm{~Hz}), 3.92(\mathrm{~s}$, $3 \mathrm{H}), 3.66(\mathrm{t}, 1 \mathrm{H}, J=6.9 \mathrm{~Hz}), 3.49-3.43(\mathrm{~m}, 1 \mathrm{H}), 2.57(\mathrm{dd}, 1 \mathrm{H}, J=17.3,6.1 \mathrm{~Hz}), 1.94(\mathrm{dd}, 1 \mathrm{H}, J$ $=17.3,7.5 \mathrm{~Hz}), 1.41(\mathrm{~s}, 9 \mathrm{H}) ;{ }^{13} \mathrm{C} \mathrm{NMR}\left(100 \mathrm{MHz}, \mathrm{CDCl}_{3}\right) \delta 171.0,169.0,164.3,136.9,131.4$, $129.2,128.9,128.0,127.8,124.5,114.2,113.6,81.1,56.0,44.2,41.9,32.5,28.2$; IR (thin film) $v$ 3086, 2976, 1715, 1594, 1496, 1364, 1264, 1167, $1080 \mathrm{~cm}^{-1}$; HRMS (ESI) calcd for $\mathrm{C}_{24} \mathrm{H}_{27} \mathrm{NO}_{6} \mathrm{~S}$ $[\mathrm{M}+\mathrm{Na}]^{+} 480.1451$, found $480.1456 ; 97 \%$ ee $(3 S, 4 S)$-isomer as determined by HPLC (AS-H, 9:1 hexanes/i-PrOH), $t_{\mathrm{r}}(3 S, 4 S)=19.4 \mathrm{~min}, t_{\mathrm{r}}(3 R, 4 R)=14.1 \mathrm{~min}$.

From (R,S) Catalyst:

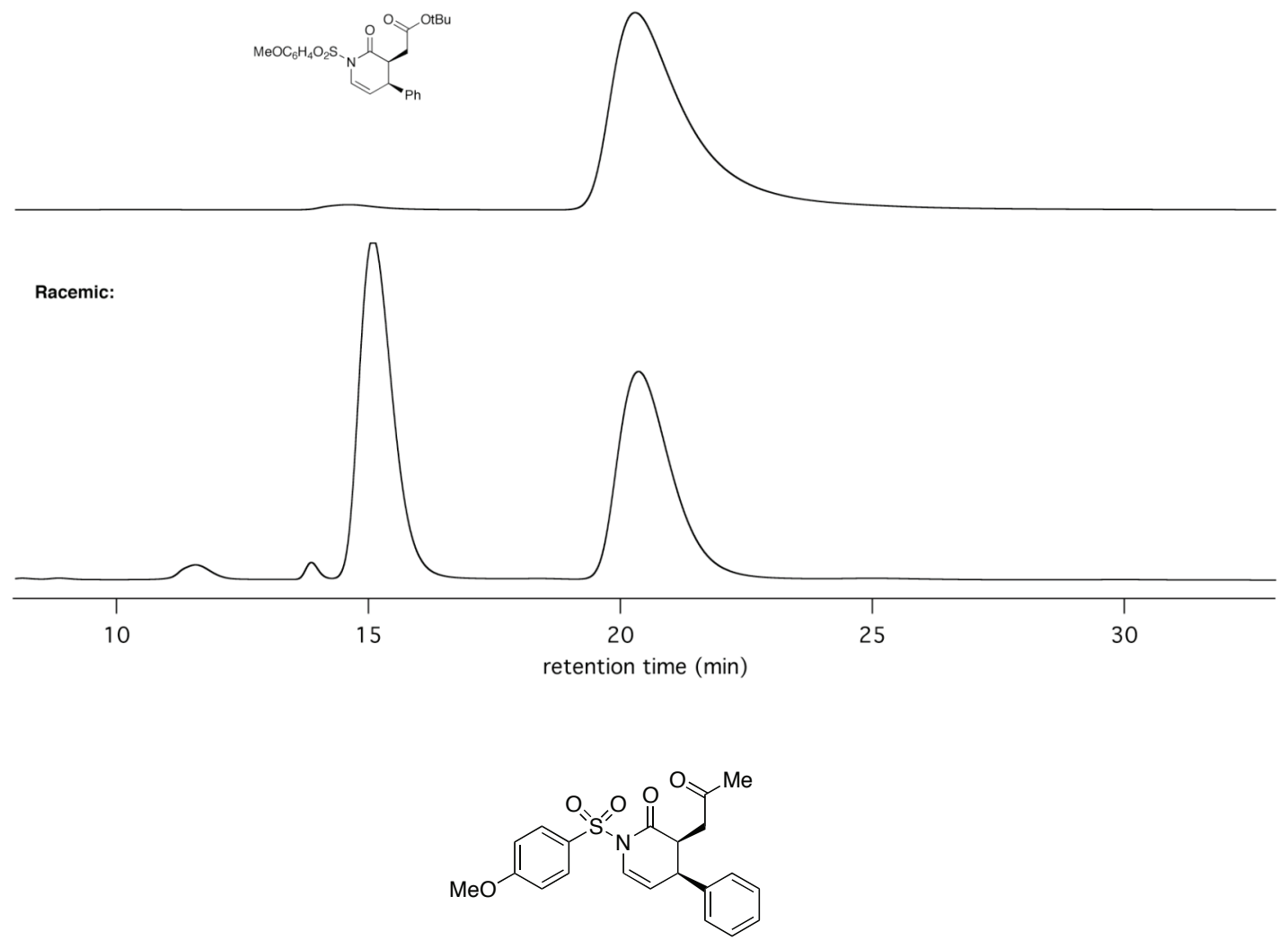

\section{(3S,4S)-1-(4-Methoxyphenylsulfonyl)-3-(2-oxopropyl)-4-phenyl-3,4-dihydropyridin-2(1H)-}

one (Table 3, entry 2). Into an oven dried $2.0 \mathrm{~mL}$ vial was added (E)-4-oxopent-2-enal (10.0 mg $0.10 \mathrm{mmol}, 1.0$ equiv), trans- $N$-(4-methoxybenzenesulfonyl)-3-phenylprop-2-en-1-imine (30.1 $\mathrm{mg}, 0.10 \mathrm{mmol}, 1.0$ equiv) and chiral catalyst 9 (5.5 mg, $0.01 \mathrm{mmol}, 0.10$ equiv). The vial was 
crimped with a Teflon-lined crimp seal. To this mixture was added $2.0 \mathrm{~mL} \mathrm{10:1} \mathrm{toluene/THF}$ $(0.05 \mathrm{M})$, followed by diisopropylethylamine $(1.7 \mu \mathrm{L}, 0.01 \mathrm{mmol}, 0.10$ equiv). The resulting solution was stirred at room temperature for $15 \mathrm{~h}$ before an additional 1.0 equiv of enal was added and the resulting solution was stirred for an additional $23 \mathrm{~h}$. The reaction mixture was concentrated under reduced pressure, and the residue was purified by PTLC (2:1 hexane/EtOAc) to afford the lactam product as a white solid $(20.4 \mathrm{mg}, 51 \%$ yield $)$. $[\alpha]_{\mathrm{D}}{ }^{20}\left(\mathrm{c} 1.10, \mathrm{CHCl}_{3}\right)=$ +186.4; $\mathrm{mp}=150-152{ }^{\circ} \mathrm{C} ;{ }^{1} \mathrm{H} \mathrm{NMR}\left(400 \mathrm{MHz}, \mathrm{CDCl}_{3}\right) \delta 7.97(\mathrm{dd}, 2 \mathrm{H}, J=6.9,2.0 \mathrm{~Hz}), 7.14(\mathrm{~d}$, $2 \mathrm{H}, J=8.2 \mathrm{~Hz}), 7.06-6.98(\mathrm{~m}, 5 \mathrm{H}), 6.56(\mathrm{dd}, 2 \mathrm{H}, J=7.2,1.1 \mathrm{~Hz}), 5.60(\mathrm{dd}, 1 \mathrm{H}, J=8.0,6.2$ Hz), 3.93 (s, 3H), 3.62-3.60 (m, 2H), 2.75 (dd, 1H, J = 18.6, 5.2 Hz), 2.05 (s, 3H), 2.04 (dd, 1H, $J=18.6,6.9 \mathrm{~Hz}) ;{ }^{13} \mathrm{C} \mathrm{NMR}\left(100 \mathrm{MHz}, \mathrm{CDCl}_{3}\right) \delta 206.3,169.5,164.3,137.4,131.4,129.0,127.8$, 124.4, 114.3, 113.7, 56.0, 43.0, 41.8, 40.1, 30.5; IR (thin film) v 3111, 3028, 2842, 1713, 1594, 1496, 1362, 1264, 1167, 1091, $1024 \mathrm{~cm}^{-1}$; HRMS (ESI) calcd for $\mathrm{C}_{21} \mathrm{H}_{21} \mathrm{NO}_{5} \mathrm{~S}[\mathrm{M}+\mathrm{Na}]^{+}$ 422.1032, found 422.1034; >99\% ee $(3 S, 4 S)$-isomer as determined by HPLC (AD-H, 9:1 hexanes/i-PrOH $), t_{\mathrm{r}}(3 S, 4 S)=44.3 \mathrm{~min}, t_{\mathrm{r}}(3 R, 4 R)=36.2 \mathrm{~min}$.

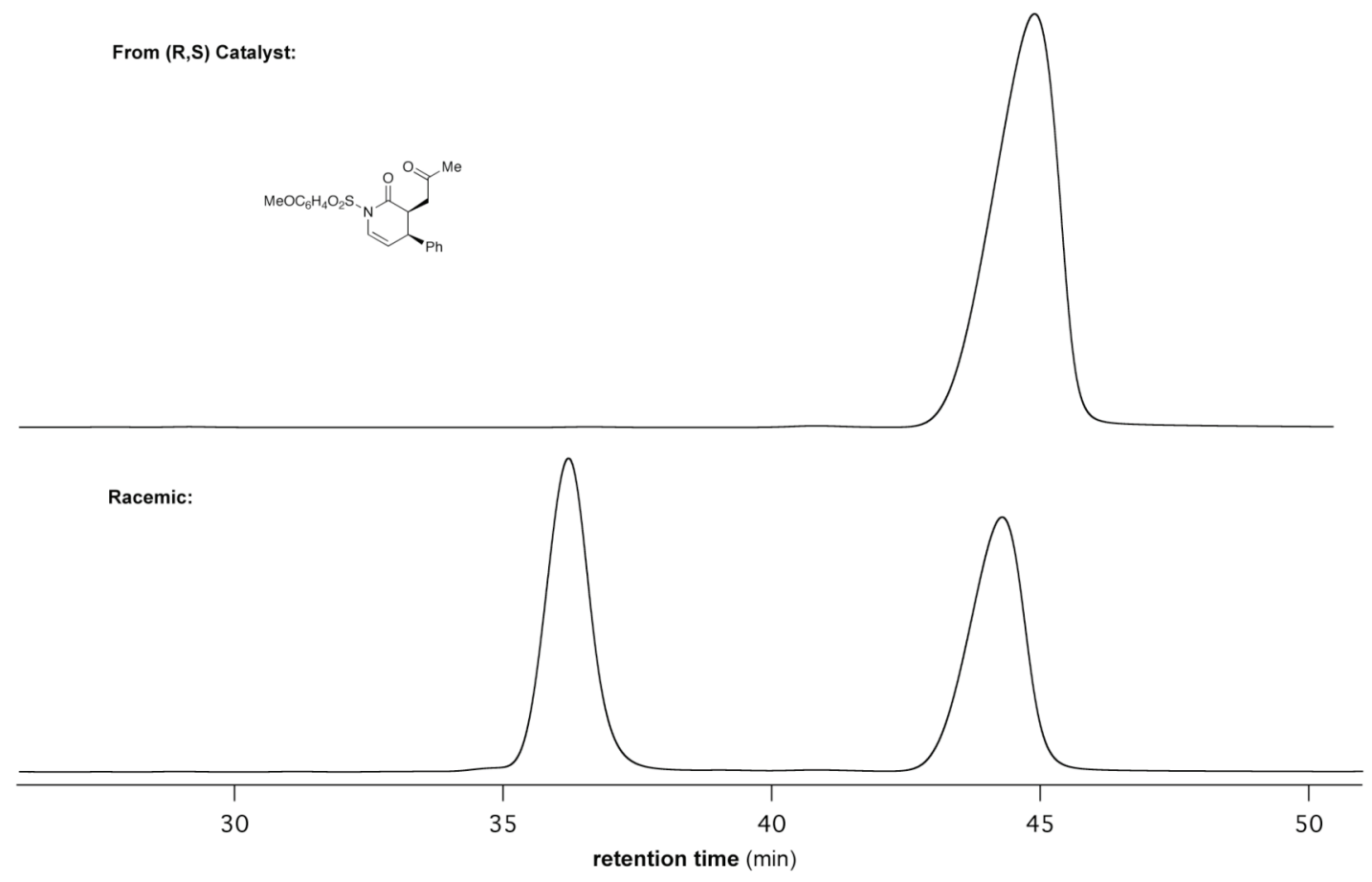




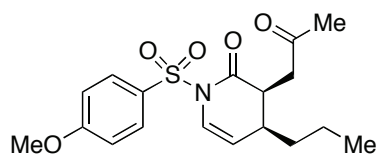

\section{(3S,4S)-1-(4-Methoxyphenylsulfonyl)-3-(2-oxopropyl)-4-propyl-3,4-dihydropyridin-2(1H)-}

one (Table 3, entry 3). Prepared according to the general procedure from $(E)$-4-oxopent-2-enal and trans- $N$-(4-methoxybenzenesulfonyl)-hex-2-en-1-imine using $10 \mathrm{~mol} \% \mathbf{9}$ as the catalyst in $70 \%$ yield as a yellow oil. $[\alpha]_{\mathrm{D}}{ }^{20}\left(\mathrm{c} 0.91, \mathrm{CHCl}_{3}\right)=-5.7 ;{ }^{1} \mathrm{H} \mathrm{NMR}\left(400 \mathrm{MHz}, \mathrm{CDCl}_{3}\right) \delta 7.91$ (dd, $2 \mathrm{H}, J=6.9,2.0 \mathrm{~Hz}), 6.97(\mathrm{dd}, 2 \mathrm{H}, J=6.9,2.0 \mathrm{~Hz}), 5.54(\mathrm{dd}, 1 \mathrm{H}, J=8.0,6.6 \mathrm{~Hz}), 3.87(\mathrm{~s}, 3 \mathrm{H})$, $3.24(\mathrm{q}, 1 \mathrm{H}, J=6.3 \mathrm{~Hz}), 2.82(\mathrm{dd}, 1 \mathrm{H}, J=16.6,7.2 \mathrm{~Hz}), 2.41-2.38(\mathrm{~m}, 1 \mathrm{H}), 2.24(\mathrm{dd}, 1 \mathrm{H}, J=$ 16.6, 7.0 Hz), $1.22(\mathrm{t}, 3 \mathrm{H}, J=7.2 \mathrm{~Hz}), 1.25-1.02(\mathrm{~m}, 4 \mathrm{H}), 0.73(\mathrm{t}, 3 \mathrm{H}, J=7.2 \mathrm{~Hz}) ;{ }^{13} \mathrm{C} \mathrm{NMR}$ $\left(100 \mathrm{MHz}, \mathrm{CDCl}_{3}\right) \delta 206.0,170.7,164.1,131.0,129.6,128.8,124.1,115.1,114.4,114.1,55.9$, 42.4, 39.8, 34.7, 32.1, 30.6, 19.5, 14.3; IR (thin film) v 3010, 2957, 1713, 1595, 1497, 1362, 1262, 1092, $1023 \mathrm{~cm}^{-1}$; HRMS (ESI) calcd for $\mathrm{C}_{18} \mathrm{H}_{23} \mathrm{NO}_{5} \mathrm{~S}[\mathrm{M}+\mathrm{Na}]^{+} 388.1189$, found 388.1175; 98\% ee $(3 S, 4 S)$-isomer as determined by HPLC (AS-H, 9:1 hexanes/i-PrOH), $t_{\mathrm{r}}(3 S, 4 S)=68.1$ $\min , t_{\mathrm{r}}(3 R, 4 R)=55.1 \min$.

From $(\mathbf{R}, \mathbf{S})$ catalyst:
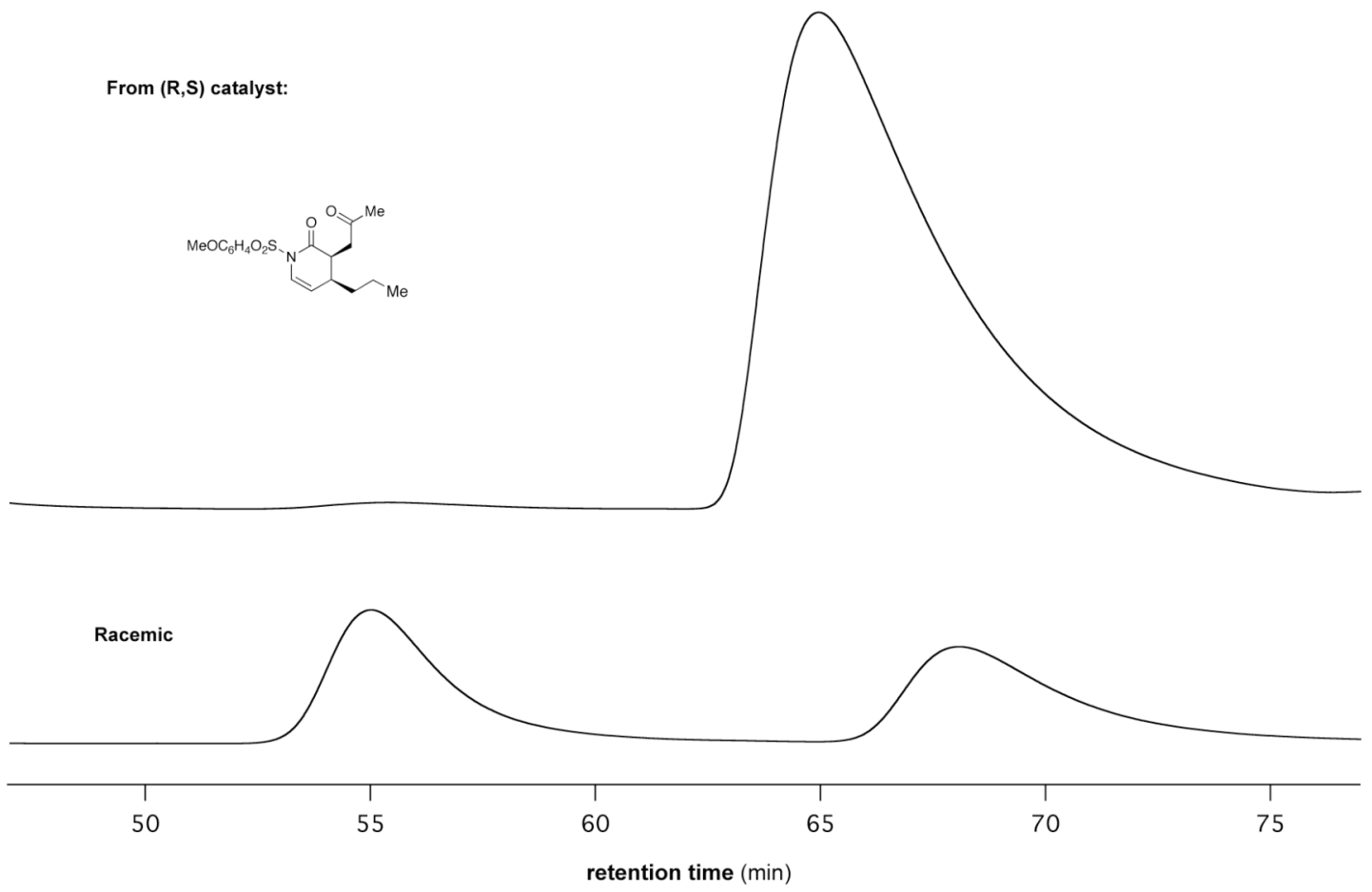


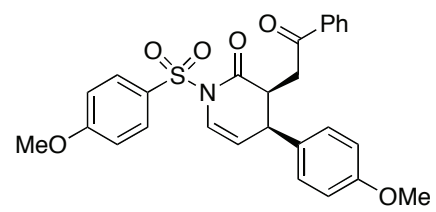

\section{(3S,4S)-4-(4-Methoxyphenyl)-1-(4-methoxyphenylsulfonyl)-3-(2-oxophenethyl)-3,4-}

dihydropyridin-2(1H)-one (Table 3, entry 4). Prepared according to the general procedure from (E)-4-oxo-4-phenylbut-2-enal and trans- $N$-(4-methoxybenzenesulfonyl)-3-(4methoxyphenyl)prop-2-en-1-imine using $10 \mathrm{~mol} \% \mathbf{9}$ as the catalyst in 52\% yield as a yellow solid. $[\alpha]_{\mathrm{D}}{ }^{20}\left(\mathrm{c} 0.87, \mathrm{CHCl}_{3}\right)=+232.3 ; \mathrm{mp}=159-161{ }^{\circ} \mathrm{C} ;{ }^{1} \mathrm{H}$ NMR $\left(400 \mathrm{MHz}, \mathrm{CDCl}_{3}\right) \delta 8.06$ $(\mathrm{dd}, 2 \mathrm{H}, J=7.0,2.0 \mathrm{~Hz}), 7.81(\mathrm{dd}, 1 \mathrm{H}, J=7.2,1.4 \mathrm{~Hz}), 7.55-7.51(\mathrm{~m}, 1 \mathrm{H}), 7.41-7.38(\mathrm{~m}, 1 \mathrm{H})$, $7.17(\mathrm{~d}, 1 \mathrm{H}, J=8.1 \mathrm{~Hz}), 7.06(\mathrm{dd}, 2 \mathrm{H}, J=7.0,2.0 \mathrm{~Hz}), 6.51-6.45(\mathrm{~m}, 4 \mathrm{H}), 5.64(\mathrm{dd}, 1 \mathrm{H}, J=$ 8.1, $6.3 \mathrm{~Hz}), 3.92$ (s, 3H), 3.90- 3.74 (m, 2H), 3.70 (s, 3H), 3.37 (dd, 1H, $J=18.4,3.5 \mathrm{~Hz}), 2.61$ $(\mathrm{dd}, 1 \mathrm{H}, J=18.4,8.0 \mathrm{~Hz}) ;{ }^{13} \mathrm{C} \mathrm{NMR}\left(100 \mathrm{MHz}, \mathrm{CDCl}_{3}\right) \delta 197.8,169.8,164.3,159.1,136.7$, $133.5,131.4,129.3,129.1,128.9,128.8,128.2$, 124.2, 114.3, 114.3, 114.2, 56.0, 55.3, 43.5, 40.7, 35.1; IR (thin film) v 3025, 2987, 1716, 1684, 1495, 1510, 1361, 1264, 1166, 1091, $1028 \mathrm{~cm}^{-1}$; HRMS (ESI) calcd for $\mathrm{C}_{27} \mathrm{H}_{25} \mathrm{NO}_{6} \mathrm{~S}[\mathrm{M}+\mathrm{Na}]^{+} 514.1294$, found 514.1307 ; >99\% ee $(3 S, 4 S)$ isomer as determined by HPLC (AS-H, 4:1 hexanes/i-PrOH), $t_{\mathrm{r}}(3 S, 4 S)=64.7$ min, $t_{\mathrm{r}}(3 R, 4 R)=$ $52.2 \mathrm{~min}$.

From $(\mathbf{R}, \mathbf{S})$ catalyst:
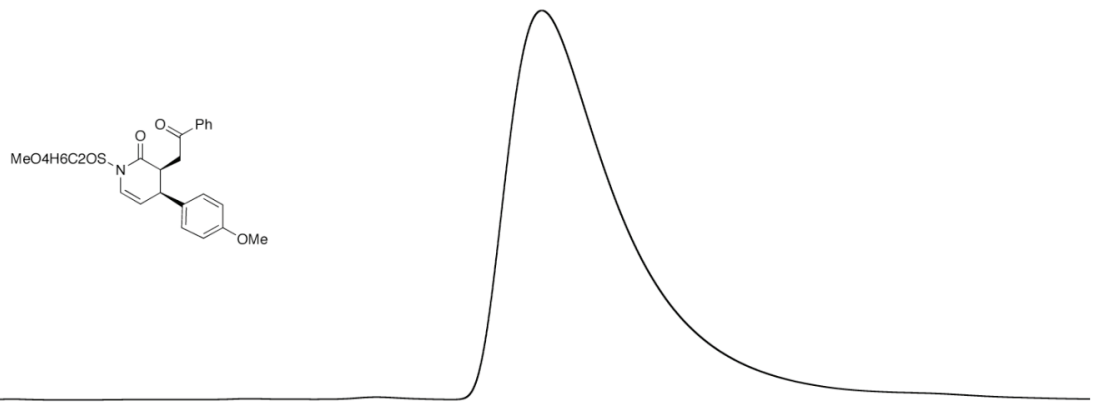

Racemic

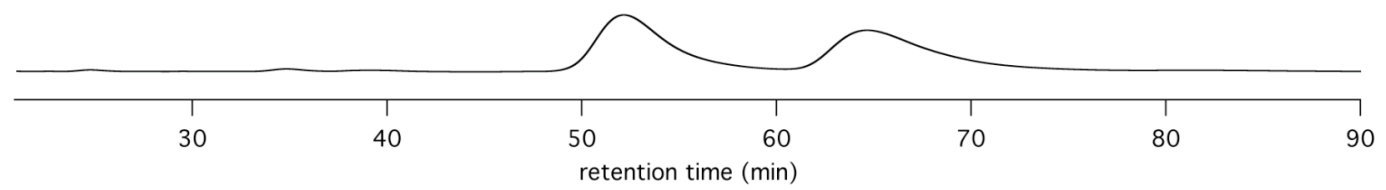

Page S11 


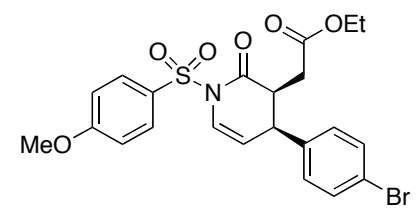

Ethyl 2-((3S,4S)-4-(4-bromophenyl)-1-(4-methoxyphenylsulfonyl)-2-oxo-1,2,3,4-tetrahydropyridin-3-yl)acetate. Prepared according to the general procedure from ethyl trans-4-oxo-2butenoate and trans- $N$-(4-methoxybenzenesulfonyl)-3-(4-bromophenyl)prop-2-en-1-imine in $35 \%$ yield as a yellow solid. $[\alpha]_{\mathrm{D}}{ }^{20}\left(\mathrm{c} 1.42, \mathrm{CHCl}_{3}\right)=+151.4 ; \mathrm{mp}=109-111{ }^{\circ} \mathrm{C} ;{ }^{1} \mathrm{H} \mathrm{NMR}(400$ $\left.\mathrm{MHz}, \mathrm{CDCl}_{3}\right) \delta 8.02(\mathrm{dd}, 2 \mathrm{H}, J=7.1,2.1 \mathrm{~Hz}), 7.17-7.12(\mathrm{~m}, 2 \mathrm{H}), 7.05(\mathrm{dd}, 2 \mathrm{H}, J=7.1,2.2 \mathrm{~Hz})$, $6.46(\mathrm{dd}, 2 \mathrm{H}, J=6.5,2.1 \mathrm{~Hz}), 5.57(\mathrm{dd}, 1 \mathrm{H}, J=8.0,6.6 \mathrm{~Hz}), 4.14-4.10(\mathrm{~m}, 2 \mathrm{H}), 3.94(\mathrm{~s}, 3 \mathrm{H})$, 3.66-3.60 (m, 1H), 3.53- $3.51(\mathrm{~m}, 1 \mathrm{H}), 2.59(\mathrm{dd}, 1 \mathrm{H}, J=17.4,6.0 \mathrm{~Hz}), 1.91$ (dd, 1H, $J=17.4$, $7.9 \mathrm{~Hz}), 1.22(\mathrm{t}, 3 \mathrm{H}, J=7.4 \mathrm{~Hz}) ;{ }^{13} \mathrm{C} \mathrm{NMR}\left(100 \mathrm{MHz}, \mathrm{CDCl}_{3}\right) \delta 171.6,168.7,164.4,135.8,132.1$ 131.4, 129.6, 128.8, 125.0, 121.8, 114.4, 114.4, 113.0, 61.1, 56.1, 43.9, 41.3, 31.4, 14.3; IR (thin film) $v 3087,2978,1719,1594,1496,1365,1263,1190,1166,1091,1025 \mathrm{~cm}^{-1}$; HRMS (ESI) calcd for $\mathrm{C}_{22} \mathrm{H}_{22} \mathrm{BrNO}_{6} \mathrm{~S}[\mathrm{M}+\mathrm{Na}]^{+}$530.0248, found 530.0254; >99\% ee $(3 S, 4 S)$-isomer as determined by HPLC (AS-H, 9:1 hexanes/i-PrOH), $t_{\mathrm{r}}(3 S, 4 S)=65.6 \mathrm{~min}, t_{\mathrm{r}}(3 R, 4 R)=44.6 \mathrm{~min}$.

From $(\mathbf{R}, \mathbf{S})$ Catalyst:
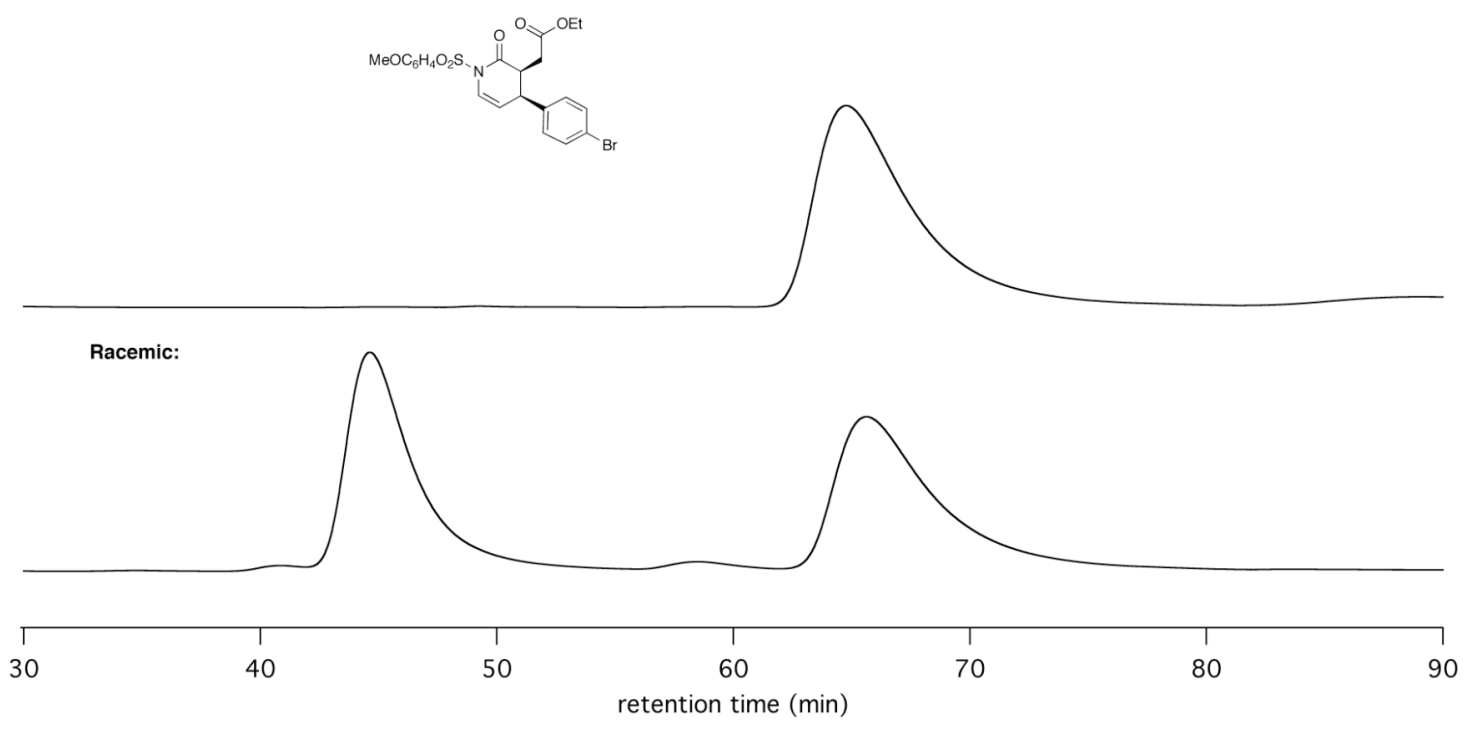

Page S12 


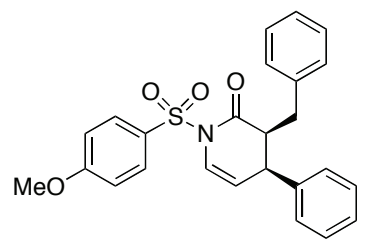

cis-3-Benzyl-1-(4-methoxyphenylsulfonyl)-4-phenyl-3,4-dihydropyridin-2(1H)-one

(14).

Prepared according to the general procedure from 2-chloro-3-phenylpropanal and trans- $\mathrm{N}$-(4methoxybenzenesulfonyl)-3-phenyprop-2-ene-1-imine using $10 \mathrm{~mol} \% 7$ and 1.5 equiv DIPEA in $40 \%$ yield. ${ }^{1} \mathrm{H}$ NMR (400 MHz, $\left.\mathrm{CDCl}_{3}\right) \delta 8.02(\mathrm{dd}, 2 \mathrm{H}, J=7.0,2.1 \mathrm{~Hz}), 7.24-7.10(\mathrm{~m}, 4 \mathrm{H})$, 7.09- $6.94(\mathrm{~m}, 7 \mathrm{H}), 6.47(\mathrm{dd}, 2 \mathrm{H}, J=8.0,1.0 \mathrm{~Hz}), 5.48(\mathrm{dd}, 1 \mathrm{H}, J=8.0,6.3 \mathrm{~Hz}), 3.90(\mathrm{~s}, 3 \mathrm{H})$, 3.33- $3.30(\mathrm{~m}, 1 \mathrm{H}), 3.27-3.21(\mathrm{~m}, 1 \mathrm{H}), 3.15(\mathrm{dd}, 1 \mathrm{H}, J=14.6,4.3 \mathrm{~Hz}), 2.10$ (dd, $1 \mathrm{H}, J=14.6$, $9.0 \mathrm{~Hz}) ;{ }^{13} \mathrm{C}$ NMR $\left(100 \mathrm{MHz}, \mathrm{CDCl}_{3}\right) \delta 170.2,164.3,138.8,137.4,131.5,129.2,129.1,128.8$, 128.7, 128.2, 127.7, 126.6, 124.1, 114.4, 114.3, 56.0, 48.9, 41.1, 31.5; IR (thin film) v 3062, 3029, 2926, 1721, 1594, 1496, 1362, 1263, 1167, 1091, $1027 \mathrm{~cm}^{-1}$; HRMS(ESI) calcd for $\mathrm{C}_{25} \mathrm{H}_{23} \mathrm{NO}_{4} \mathrm{~S}[\mathrm{M}+\mathrm{Na}]^{+} 456.1240$, found 456.1247 .

\section{Preparation of Enals.}

All enals were prepared according to the literature procedures and have been previously reported $^{2,3}$.

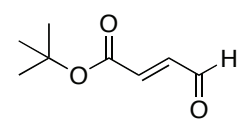

(E)-tert-Butyl 4-oxobut-2-enoate. ${ }^{1} \mathrm{H}$ NMR (400 MHz, $\left.\mathrm{CDCl}_{3}\right) \delta 9.58(\mathrm{~d}, 1 \mathrm{H}, J=7.4 \mathrm{~Hz}), 6.71$ $(\mathrm{dd}, 1 \mathrm{H}, J=15.9,7.4 \mathrm{~Hz}), 6.51(\mathrm{~d}, 1 \mathrm{H}, J=15.9 \mathrm{~Hz}), 1.36(\mathrm{~s}, 12 \mathrm{H}) ;{ }^{13} \mathrm{C}$ NMR $\left(100 \mathrm{MHz}, \mathrm{CDCl}_{3}\right)$ ઈ 192.8, 141.7, 139.6, 82.4, 27.9 .

(2) Klei, A.; Jong, R. L. P.; Lugtenburg, J.; Tielens, A. G. M. Eur. J. Org. Chem. 2002, 3015-3023.

(3) Satoh, T.; Hanaki, N.; Kuramochi, Y.; Inoue, Y.; Hosoya, K.; Sakai, K. Tetrahedron, 2002, 58, 2533-2549. 


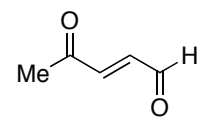

(E)-4-Oxopent-2-enal. ${ }^{1} \mathrm{H}$ NMR $\left(400 \mathrm{MHz}, \mathrm{CDCl}_{3}\right) \delta 9.77(\mathrm{~d}, 1 \mathrm{H}, J=7.1 \mathrm{~Hz}), 6.83(\mathrm{~d}, 1 \mathrm{H}, J=$ $16.3 \mathrm{~Hz}), 6.72(\mathrm{dd}, 1 \mathrm{H}, J=16.3,7.0 \mathrm{~Hz}), 2.40(\mathrm{~s}, 3 \mathrm{H}) ;{ }^{13} \mathrm{C} \mathrm{NMR}\left(100 \mathrm{MHz}, \mathrm{CDCl}_{3}\right) \delta 198.1$, $193.7,145.5,138.3,28.1$.

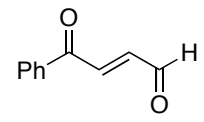

(E)-4-Oxo-4-phenylbut-2-enal. ${ }^{1} \mathrm{H}$ NMR (400 MHz, $\left.\mathrm{CDCl}_{3}\right) \delta 9.89$ (d, $1 \mathrm{H}, J=7.5 \mathrm{~Hz}$ ), 8.007.99 (m, 2H), 7.74- $7.64(\mathrm{~m}, 2 \mathrm{H}), 7.56-7.52(\mathrm{~m}, 2 \mathrm{H}), 6.99(\mathrm{dd}, 1 \mathrm{H}, J=15.8,7.5 \mathrm{~Hz}) ;{ }^{13} \mathrm{C} \mathrm{NMR}$ $\left(100 \mathrm{MHz}, \mathrm{CDCl}_{3}\right) \delta 193.0,190.0,142.3,139.4,136.4,134.4,129.2,129.2$.

General procedure for the preparation of imines. All imines were prepared according to literature protocols. ${ }^{4}$ The corresponding aldehyde dimethyl acetal (1.0 equiv) and the arylsulfonamide (1.0 equiv) were mixed in a flask equipped with a Dean-Stark condenser. The neat mixture was heated to $180{ }^{\circ} \mathrm{C}$ for $30 \mathrm{~min}$. The resulting melt was cooled to room temperature and the solid was crystallized from toluene to yield the product.

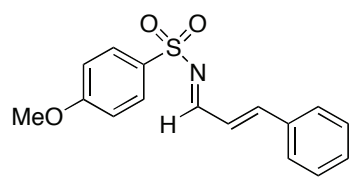

trans- $N$-(4-Methoxybenzenesulfonyl)-3-phenylprop-2-en-1-imine. ${ }^{4}{ }^{1} \mathrm{H} \quad \mathrm{NMR} \quad(400 \mathrm{MHz}$, $\left.\mathrm{CDCl}_{3}\right) \delta 8.74(\mathrm{~d}, 1 \mathrm{H}, J=9.5 \mathrm{~Hz}), 7.89(\mathrm{~d}, 2 \mathrm{H}, J=8.9 \mathrm{~Hz}), 7.54-7.40(\mathrm{~m}, 6 \mathrm{H}), 7.00-6.93(\mathrm{~m}$, 3H), 3.85 (s, 3H); ${ }^{13} \mathrm{C}$ NMR (100 MHz, $\left.\mathrm{CDCl}_{3}\right) \delta$ 170.5, 163.7, 153.8, 134.2, 131.7, 130.2, 129.7, $129.3,128.7,124.7,114.5,55.8$.

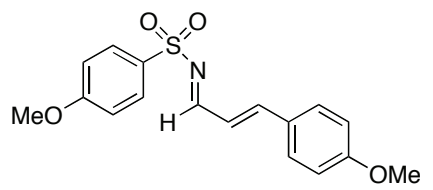

trans- $N$-(4-Methoxybenzenesulfonyl)-3-(4-methoxyphenyl)prop-2-en-1-imine. Yellow solid; $\mathrm{mp}=116-118{ }^{\circ} \mathrm{C} ;{ }^{1} \mathrm{H}$ NMR $\left(400 \mathrm{MHz}, \mathrm{CDCl}_{3}\right) \delta 8.67(\mathrm{~d}, 1 \mathrm{H}, J=9.5 \mathrm{~Hz}), 7.84(\mathrm{dd}, 2 \mathrm{H}, J=6.9$,

(4) He, M; Bode, J. W. Org. Lett. 2005, 7, 3131-3134 
$1.8 \mathrm{~Hz}), 7.46(\mathrm{~d}, 2 \mathrm{H}, J=8.8 \mathrm{~Hz}), 7.37(\mathrm{~d}, 1 \mathrm{H}, J=15.6 \mathrm{~Hz}), 6.95(\mathrm{dd}, 2 \mathrm{H}, J=6.9,1.8 \mathrm{~Hz}), 6.88$ $(\mathrm{d}, 2 \mathrm{H}, J=8.8 \mathrm{~Hz}), 6.81(\mathrm{dd}, 1 \mathrm{H}, J=15.6,9.5 \mathrm{~Hz}), 3.82(\mathrm{~s}, 3 \mathrm{H}), 3.81(\mathrm{~s}, 3 \mathrm{H}) ;{ }^{13} \mathrm{C}$ NMR $(100$ $\left.\mathrm{MHz}, \mathrm{CDCl}_{3}\right) \delta 170.9,163.7,162.7,153.9,130.8,130.2,127.2,122.5,114.9,114.6,55.9,55.7$; IR (thin film) v 3007, 2938, 2840, 1596, 1573, 1319, 1259, 1166, 1151, 1090, $1025 \mathrm{~cm}^{-1}$; HRMS (ESI) calcd for $\mathrm{C}_{17} \mathrm{H}_{17} \mathrm{NO}_{4} \mathrm{~S}[\mathrm{M}+\mathrm{Na}]^{+} 354.0770$, found 354.0766.

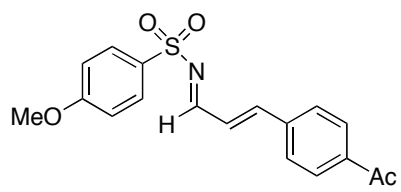

trans- $\mathrm{N}$-(4-Methoxybenzenesulfonyl)-1-(4-(3-iminoprop-1-enyl)phenyl)ethanone. Yellow solid; mp = 173-176 ${ }^{\circ} \mathrm{C} ;{ }^{1} \mathrm{H}$ NMR $\left(400 \mathrm{MHz}, \mathrm{CDCl}_{3}\right) \delta 8.72(\mathrm{~d}, 1 \mathrm{H}, J=9.3 \mathrm{~Hz}), 7.94(\mathrm{~d}, 2 \mathrm{H}, J=$ $8.3 \mathrm{~Hz}), 7.85(\mathrm{dd}, 2 \mathrm{H}, J=7.0,2.0 \mathrm{~Hz}), 7.58(\mathrm{~d}, 2 \mathrm{H}, J=8.3 \mathrm{~Hz}), 7.45(\mathrm{~d}, 1 \mathrm{H}, J=15.8 \mathrm{~Hz}), 7.00$ $(\mathrm{dd}, 1 \mathrm{H}, J=15.8,9.3 \mathrm{~Hz}), 6.96(\mathrm{dd}, 2 \mathrm{H}, J=7.0,2.0 \mathrm{~Hz}), 3.83(\mathrm{~s}, 3 \mathrm{H}), 2.57(\mathrm{~s}, 3 \mathrm{H}) ;{ }^{13} \mathrm{C}$ NMR $\left(100 \mathrm{MHz}, \mathrm{CDCl}_{3}\right) \delta 197.4,170.0,164.0,151.5,138.9,138.4,130.5,129.5,129.2,128.8,127.2$, 114.7, 114.4, 55.9, 27.0; IR (thin film) v 3009, 2840, 1682, 1623, 1579, 1498, 1266, 1151, 1091, $1023 \mathrm{~cm}^{-1}$; HRMS (ESI) calcd for $\mathrm{C}_{18} \mathrm{H}_{17} \mathrm{NO}_{4} \mathrm{~S}[\mathrm{M}+\mathrm{Na}]^{+} 366.0770$, found 366.0780.

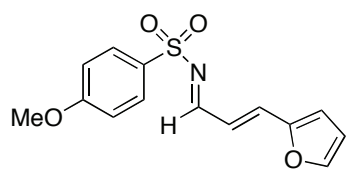

trans- $N$-(4-Methoxybenzenesulfonyl)-3-(furan-2-yl)prop-2-en-1-imine. Brown solid; $\mathrm{mp}=$ 100-103 ${ }^{\circ} \mathrm{C} ;{ }^{1} \mathrm{H}$ NMR (400 MHz, $\mathrm{CDCl}_{3}$ ) $\delta 8.67$ (d, 1H, J =9.7 Hz), 7.89 (dd, 2H, J = 6.9, 2.0 $\mathrm{Hz}), 7.57(\mathrm{~d}, 1 \mathrm{H}, J=1.9 \mathrm{~Hz}), 7.21(\mathrm{~d}, 1 \mathrm{H}, J=15.5 \mathrm{~Hz}), 7.00(\mathrm{dd}, 2 \mathrm{H}, J=6.9,2.0 \mathrm{~Hz}), 6.83(\mathrm{dd}$, $1 \mathrm{H}, J=15.5,9.7 \mathrm{~Hz}), 6.77(\mathrm{~d}, 1 \mathrm{H}, J=3.5 \mathrm{~Hz}), 6.54(\mathrm{dd}, 1 \mathrm{H}, J=3.5,1.9 \mathrm{~Hz}), 3.87(\mathrm{~s}, 3 \mathrm{H}) ;{ }^{13} \mathrm{C}$ NMR $\left(100 \mathrm{MHz}, \mathrm{CDCl}_{3}\right) \delta 170.0,163.7,151.1,146.7,138.6,130.3,128.8,122.5,117.7,114.6$, 114.4, 113.3, 55.9, 55.8; IR (thin film) v 3127, 2842, 1623, 1581, 1463, 1261, 1150, 1090, 1020 $\mathrm{cm}^{-1}$; HRMS (ESI) calcd for $\mathrm{C}_{14} \mathrm{H}_{13} \mathrm{NO}_{4} \mathrm{~S}[\mathrm{M}+\mathrm{Na}]^{+}$314.0457, found 314.0463. 


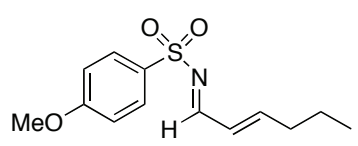

trans- $N$-(4-Methoxybenzenesulfonyl)-hex-2-en-1-imine. Yellow solid; $\mathrm{mp}=45-48{ }^{\circ} \mathrm{C} ;{ }^{1} \mathrm{H}$ NMR (400 MHz, $\left.\mathrm{CDCl}_{3}\right) \delta 8.57(\mathrm{~d}, 1 \mathrm{H}, J=9.4 \mathrm{~Hz}), 7.05(\mathrm{dd}, 2 \mathrm{H}, J=6.7,2.0 \mathrm{~Hz}), 7.00$ (dd, $2 \mathrm{H}, J=6.7,2.0 \mathrm{~Hz}), 6.87(\mathrm{dt}, 1 \mathrm{H}, J=15.4,7.0 \mathrm{~Hz}), 6.35(\mathrm{dd}, 1 \mathrm{H}, J=15.4,9.4 \mathrm{~Hz}), 3.87(\mathrm{~s}$, $3 \mathrm{H}), 2.35-2.29(\mathrm{~m}, 2 \mathrm{H}), 1.53(\mathrm{q}, 2 \mathrm{H}, J=7.3 \mathrm{~Hz}), 0.94(\mathrm{t}, 3 \mathrm{H}, J=7.4 \mathrm{~Hz}) ;{ }^{13} \mathrm{C}$ NMR $(100 \mathrm{MHz}$, $\left.\mathrm{CDCl}_{3}\right) \delta 171.0,163.7,160.6,130.2,129.6,128.5,114.5,114.5,55.8,35.6,21.2,13.7$; IR (thin film) $v 3087,2963,2934,2874,1636,1595,1498,1323,1262,1154,1092,1023 \mathrm{~cm}^{-1}$; HRMS (ESI) calcd for $\mathrm{C}_{13} \mathrm{H}_{17} \mathrm{NO}_{3} \mathrm{~S}[\mathrm{M}+\mathrm{Na}]^{+} 290.0821$, found 290.0815 .

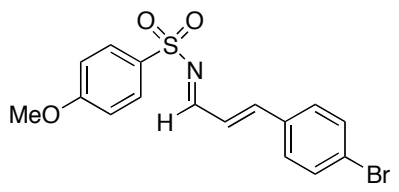

trans- $\mathrm{N}$-(4-Methoxybenzenesulfonyl)-3-(4-bromophenyl)prop-2-en-1-imine. Yellow solid; $\mathrm{mp}=170-173{ }^{\circ} \mathrm{C} ;{ }^{1} \mathrm{H}$ NMR $\left(400 \mathrm{MHz}, \mathrm{CDCl}_{3}\right) \delta 8.74(\mathrm{~d}, 1 \mathrm{H}, J=9.4 \mathrm{~Hz}), 7.90(\mathrm{~d}, 2 \mathrm{H}, J=8.9$ $\mathrm{Hz}), 7.56(\mathrm{~d}, 2 \mathrm{H}, J=8.9 \mathrm{~Hz}), 7.43-7.39(\mathrm{~m}, 3 \mathrm{H}), 7.02-6.99(\mathrm{~m}, 3 \mathrm{H}), 3.88(\mathrm{~s}, 3 \mathrm{H}) ;{ }^{13} \mathrm{C} \mathrm{NMR}$ $\left(100 \mathrm{MHz}, \mathrm{CDCl}_{3}\right) \delta 170.2,164.0,152.0,133.3,132.7,130.4,130.0,129.7,126.3,125.5,114.7$, 55.9; IR (thin film) v 3028, 2840, 1620, 1594, 1575, 1320, 1261, 1152, 1091, $1024 \mathrm{~cm}^{-1}$; HRMS (ESI) calcd for $\mathrm{C}_{16} \mathrm{H}_{14} \mathrm{BrNO}_{3} \mathrm{~S}[\mathrm{M}+\mathrm{Na}]^{+} 401.9769$, found 401.9786 .

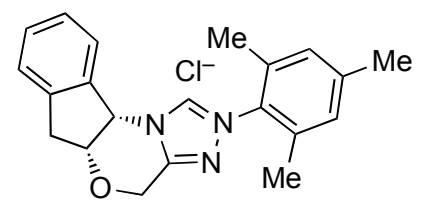

2-Mesityl-6,10b-dihydro-4H,5aH-(5R)-oxa-3,10c-diaza-(2S)-azoniacyclopenta[c]fluorene.

Prepared in analogy to the method of Rovis ${ }^{5}$ using mesitylhydrazine. White solid; $[\alpha]_{\mathrm{D}}{ }^{20}$ (c 1.00, EtOH) $=+129.3 ;{ }^{1} \mathrm{H}$ NMR (400 MHz, DMSO) $\delta 11.46(\mathrm{~s}, 1 \mathrm{H}), 7.67(\mathrm{~d}, 1 \mathrm{H}, J=7.3 \mathrm{~Hz})$, $7.45-7.33(\mathrm{~m}, 3 \mathrm{H}), 7.21(\mathrm{~s}, 2 \mathrm{H}), 6.14(\mathrm{~d}, 1 \mathrm{H}, J=3.8 \mathrm{~Hz}), 5.26(\mathrm{~d}, 1 \mathrm{H}, J=16.0 \mathrm{~Hz}), 5.08(\mathrm{~d}, 1 \mathrm{H}$, $J=16.0 \mathrm{~Hz}), 4.99(\mathrm{t}, 1 \mathrm{H}, J=4.3 \mathrm{~Hz}), 3.49(\mathrm{dd}, 1 \mathrm{H}, J=4.9 \mathrm{~Hz}, 17.1 \mathrm{~Hz}), 3.16(\mathrm{~d}, 1 \mathrm{H}, J=17.1$

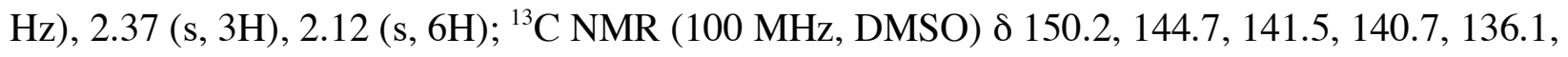

(5) Kerr, M. S.; Read de Alaniz, J.; Rovis, T. J. Org. Chem. 2005, 70, 5725-5728. 
134.9, 131.3, 129.4, 129.3, 127.3, 125.5, 124.0, 76.9, 61.2, 59.8, 37.1, 20.7, 17.0; IR (thin film) $v$ 2923, 2853, 1580, 1460, 1222, $1099 \mathrm{~cm}^{-1}$; HRMS (ESI) calcd for $\mathrm{C}_{21} \mathrm{H}_{22} \mathrm{~N}_{3} \mathrm{O}^{+}[\mathrm{M}]^{+} 332.1757$, found 332.1754. For the $(S, R)$-enantiomer: $\quad[\alpha]_{\mathrm{D}}{ }^{20}(\mathrm{c} 1.00, \mathrm{EtOH})=-129.4$.

\section{Determination of the relative and absolute stereochemistry of the dihydropyridinone}

products. The stereochemistry was determined by single crystal x-ray analysis of the product derived from 4-bromo-cinnamaldehyde imine (see above for characterization detais.): A colorless crystal of approximate dimensions $0.3 * 0.2 * 0.09 \mathrm{~mm}$ was mounted on a glass fiber and transferred to a Bruker CCD platform diffractometer. The SMART ${ }^{6}$ program was used to determine the unite cell parameters and data collection $(20 \mathrm{sec} /$ frame, $0.3 \mathrm{deg}$. /frame for a sphere of diffraction data). The data were collected at room temperature. The raw frame data were processed using $\mathrm{SAINT}^{7}$ program. The absorption correction was applied using program SADABS. $^{8}$ The structure was solved by direct methods and refined on F2 by full-matrix leastsquares techniques. Hydrogen atoms were theoretically added. At convergence, GOF = 1.099 for 288 variables refined to $\mathrm{R} 1=0.0822$ for 1517 reflections with $\mathrm{I}>2 \sigma(\mathrm{I})$. The absolute structure was determined from the Flack parameters, ${ }^{9}$ the two enantiomers gave the Flack parameters 0.03(2) and 1.04(0.03), respectively. See the corresponding CIF file for further information.
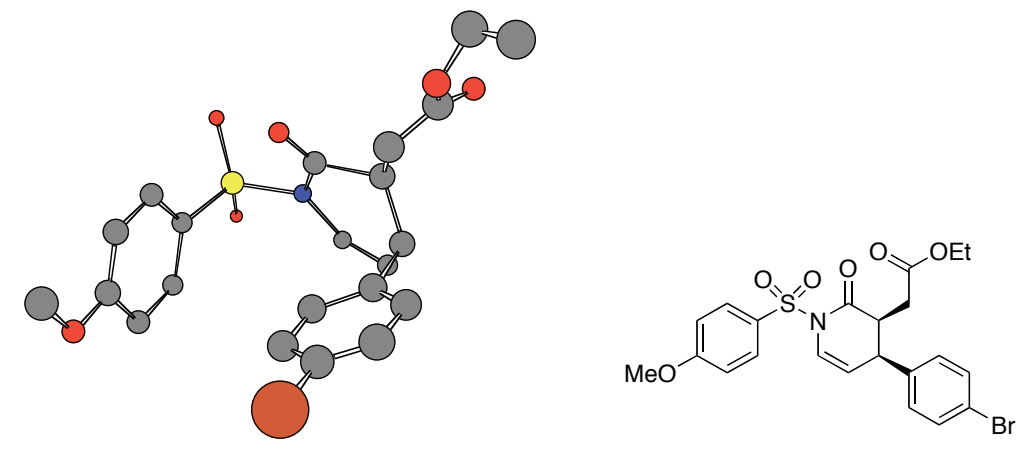

(6) SMART Software Users Guide, Version 5.1, Bruker Analytical X-ray Systems, Inc., Madison, WI 1999.

(7) SAINT Software Users Guide, Version 5.1, Bruker Analytical X-ray Systems, Inc., Madison, WI 1999.

(8) Sheldrick, G. M. SADABS, Version 2.05, Bruker Analytical X-ray Systerms, Inc.; Madison, WI 2001.

(9) Flack, H. D., Acta Cryst. A39(1983) 876-881. 


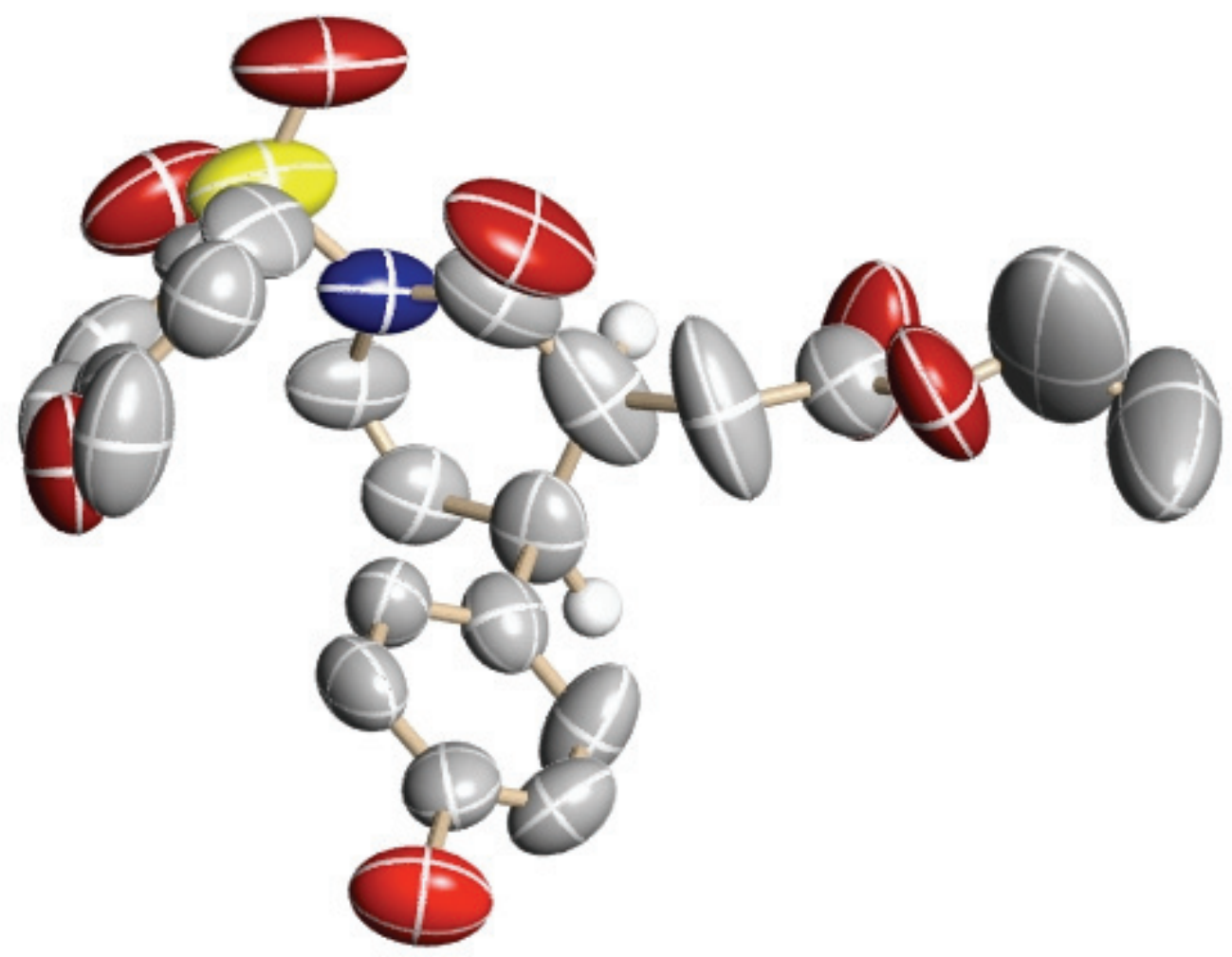

Figure 1. ORTEP representation (50\% probability level) of ethyl 2-((3S,4S)-4-(4bromophenyl)-1-(4-methoxyphenylsulfonyl)-2-oxo-1,2,3,4-tetrahydro-pyridin-3-yl)acetate. For clarity only the $\mathrm{H}$ atoms at the stereogenic centers are shown. 